Asia Pacific Journal of Mathematics, Vol. 5, No. 1 (2018), 132-138

ISSN 2357-2205

\title{
MAJORIZATION PROBLEM FOR CERTAIN CLASSES OF ANALYTIC FUNCTIONS DEFINED BY GENERALIZED OPERATOR
}

\author{
H. E. DARWISH ${ }^{1}$, A.Y. LASHIN ${ }^{1}$, R.M. EL-ASHWAH ${ }^{2}$ AND E. M. MADAR ${ }^{2, *}$ \\ ${ }^{1}$ Department of Mathematics Faculty of Science, Mansoura University Mansoura, 35516, EGYPT \\ ${ }^{2}$ Department of Mathematics Faculty of Science, Damietta University, New Damietta, 34517, EGYPT \\ ${ }^{*}$ Corresponding author: entesarmadar@gmail.com
}

Received Jan 2, 2018

\begin{abstract}
In this paper, we investigate majorization properties for certain classes of multivalent analytic functions defined by a generalized operator. Also, we point out some new and known consequences of our main result.
\end{abstract}

2010 Mathematics Subject Classification. 30C45.

Key words and phrases. rational functions; starlike functions; convex functions; subordination; Majorization.

\section{INTRODUCTION}

Let $\mathcal{A}_{p}$ denote the class of functions $f(z)$ of the form

$$
f(z)=z^{p}+\sum_{k=1}^{\infty} a_{k} z^{p+k}, \quad(p \in \mathbb{N}:=\{1,2,3, \ldots\},
$$

which are analytic and p-valent in the open unit disk $\Delta=\{z: z \in \mathbb{C}$ and $|z|<1\}$. Also, let $\mathcal{A}_{1}:=\mathcal{A}$. For functions $f_{j} \in \mathcal{A}_{p}$ given by

$$
f_{j}(z)=z^{p}+\sum_{k=1}^{\infty} a_{k, j} z^{p+k}, \quad(j=1,2 ; p \in \mathbb{N})
$$

we define the Hadamard product (or convolution) of $f_{1}$ and $f_{2}$ by

$$
\left(f_{1} * f_{2}\right)(z)=z^{p}+\sum_{k=1}^{\infty} a_{k, 1} a_{k, 2} z^{p+k}=\left(f_{2} * f_{1}\right)(z) .
$$

Let $f(z)$ and $g(z)$ be analytic in $\Delta$. Then we say that the function $f(z)$ is subordinate to $g(z)$ in $\Delta$, if there exists an analytic function $w(z)$ in $\Delta$ with

$$
w(0)=0, \quad|w(z)|<1 \quad(z \in \Delta),
$$

such that

$$
f(z)=g(w(z)) \quad(z \in \Delta) .
$$

(c)2018 Asia Pacific Journal of Mathematics 
We denote this subordination by $f(z) \prec g(z)$. Furthermore, if the function $g(z)$ is univalent in $\Delta$, then $f(z) \prec g(z)(z \in \Delta) \Leftrightarrow f(0)=g(0)$ and $f(\Delta) \subset g(\Delta)$.

Suppose that the functions $f(z)$ and $g(z)$ are analytic in open unit disk $\Delta$. Then we say that the function $f(z)$ is majorized by $g(z)$ in $\Delta$ (see [5]) and written as follows

$$
f(z) \ll g(z) \quad(z \in \Delta)
$$

if there exists a function $\phi(z)$, analytic in $\Delta$, such that

$$
|\phi(z)| \leq 1 \text { and } f(z)=\phi(z) g(z) \quad(z \in \Delta) .
$$

The majorization (1.3) is closely related to the concept of quasi-subordination between analytic functions in $\Delta$.

Let $\alpha_{1}, \alpha_{2}, \ldots, \alpha_{q}$ and $\beta_{1}, \beta_{2}, \ldots, \beta_{s}(q, s \in \mathbb{N} \cup\{0\}, q \leq s+1)$ be complex numbers such that $\beta_{\ell} \neq 0,-1,-2, \ldots$ for $\ell \in\{1,2, \ldots, s\}$. The generalized hypergeometric function ${ }_{q} F_{s}$ is given by

$$
{ }_{q} F_{s}\left(\alpha_{1}, \alpha_{2}, \ldots, \alpha_{q} ; \beta_{1}, \beta_{2}, \ldots, \beta_{s} ; z\right)=\sum_{k=0}^{\infty} \frac{\left(\alpha_{1}\right)_{k},\left(\alpha_{2}\right)_{k}, \ldots,\left(\alpha_{q}\right)_{k}}{\left(\beta_{1}\right)_{k},\left(\beta_{2}\right)_{k}, \ldots,\left(\beta_{s}\right)_{k}} \frac{z^{n}}{n !}, \quad(z \in \Delta),
$$

where $(x)_{k}$ denotes the pochhammer symbol defined by

$$
(x)_{k}=x(x+1)(x+2) \ldots(x+n-1) \text { for } n \in \mathbb{N} \text { and }(x)_{0}=1 .
$$

Corresponding to a function $\Re_{q, s}\left(\alpha_{1}, \beta_{1} ; z\right)$ defined by

$$
\Re_{q, s}\left(\alpha_{1}, \beta_{1} ; z\right):=z^{p}{ }_{q} F_{s}\left(\alpha_{1}, \alpha_{2}, \ldots, \alpha_{q} ; \beta_{1}, \beta_{2}, \ldots, \beta_{s} ; z\right),
$$

Selvaraj and Selvakumarn [10] recently defined the following generalized differential operator $G_{\lambda}^{p, m}\left(\alpha_{1}, \beta_{1}\right) f: \mathcal{A}_{p} \rightarrow \mathcal{A}_{p}$ by

$$
\begin{gathered}
G_{\lambda}^{p, 0}\left(\alpha_{1}, \beta_{1}\right) f(z)=f(z) * G_{q, s}^{p}\left(\alpha_{1}, \beta_{1} ; z\right) \\
G_{\lambda}^{p, 1}\left(\alpha_{1}, \beta_{1}\right) f(z)=(1-\lambda)\left(f(z) * G_{q, s}^{p}\left(\alpha_{1}, \beta_{1} ; z\right)\right)+\frac{\lambda}{p} z\left(f(z) * G_{q, s}^{p}\left(\alpha_{1}, \beta_{1} ; z\right)\right)^{\prime} \\
G_{\lambda}^{p, m}\left(\alpha_{1}, \beta_{1}\right) f(z)=G_{\lambda}^{p, 1}\left(G_{\lambda}^{p, m-1}\left(\alpha_{1}, \beta_{1}\right) f(z)\right),
\end{gathered}
$$

where $m \in \mathbb{N}_{0}=\mathbb{N} \cup\{0\}$ and $\lambda \geq 0$.

If $f(z) \in \mathcal{A}_{p}$, then we have

$$
G_{\lambda}^{p, m}\left(\alpha_{1}, \beta_{1}\right) f(z)=z^{p}+\sum_{k=1}^{\infty}\left(\frac{p+\lambda k}{p}\right)^{m} \frac{\left(\alpha_{1}\right)_{k},\left(\alpha_{2}\right)_{k}, \ldots,\left(\alpha_{q}\right)_{k}}{\left(\beta_{1}\right)_{k},\left(\beta_{2}\right)_{k}, \ldots,\left(\beta_{s}\right)_{k}} a_{n} \frac{z^{p+k}}{n !} .
$$

It can be seen that, by specializing the parameters the operator $G_{\lambda}^{p, m}\left(\alpha_{1}, \beta_{1}\right) f(z)$ reduces to many known and new integral and differential operators. For example, when $m=0$ the operator $G_{\lambda}^{p, m}\left(\alpha_{1}, \beta_{1}\right) f(z)$ reduces to the well known Dziok-Srivastava operator [3] and for $p=1, q=2, s=1, \alpha_{1}=\beta_{1}$ and $\alpha_{2}=1$, it reduces to the operator introduced by AL-Oboudi 
[1]. Further we remark that, when $p=1, q=2, s=1, \alpha_{1}=\beta_{1}, \alpha_{2}=1$ and $\lambda=1$ the operator $G_{\lambda}^{p, m}\left(\alpha_{1}, \beta_{1}\right) f(z)$ reduces to the operator introduced by $G$. S. Salagean [8].

It can be easily verified from [6] that

$$
\lambda z\left(G_{\lambda}^{p, m}\left(\alpha_{1}, \beta_{1}\right) f(z)\right)^{\prime}=p G_{\lambda}^{p, m+1}\left(\alpha_{1}, \beta_{1}\right) f(z)-p(1-\lambda) G_{\lambda}^{p, m}\left(\alpha_{1}, \beta_{1}\right) f(z) .
$$

Using the operator $G_{\lambda}^{p, m}\left(\alpha_{1}, \beta_{1}\right) f(z)$ we now define the following class of p-valent analytic functions.

Definition 1. A function $f(z) \in \mathcal{A}_{p}$ is said to be in the class $M_{\lambda, m}^{p, j}(A, B ; b, \gamma)$ of p-valent functions of complex order $b \neq 0$ in $\Delta$ if and only if

$$
\operatorname{Re}\left\{1+\frac{1}{b}\left(\frac{z\left(G_{\lambda}^{p, m}\left(\alpha_{1}, \beta_{1}\right) f(z)\right)^{j+1}}{\left(G_{\lambda}^{p, m}\left(\alpha_{1}, \beta_{1}\right) f(z)\right)^{j}}-p+j\right)\right\} \prec \frac{1+[(B-A) \gamma+A] z}{1+B z}
$$

$\left(z \in \Delta ;-1 \leq B<A \leq 1,0 \leq \gamma<1 ; p \in \mathbb{N}, m, j \in \mathbb{N}_{0} ; b \in \mathbb{C}-\{0\} ; p>|b \lambda(A-B)(1-\gamma)+p B|\right)$.

It can be seen that, by specializing the parameters the class $M_{\lambda, m}^{p, j}(A, B ; b, \gamma)$ reduces to many known subclasses of analytic functions. In particular, when $\gamma=0$ the class reduces to the class $M_{\lambda, m}^{p, j}(A, B ; b)$ which has recently been introduced by Selvaraj and Selvakumaran [10] and when $A=1, B=-1$ and $\gamma=0$ the class reduces to the class $M_{\lambda, m}^{p, j}(b)$ which has recently been introduced by Selvaraj and Selvakumaran [9]. Further, when $q=2, s=1$, $\alpha_{1}=\beta_{1}$ and $\alpha_{2}=1$, we have the following relationships:

(1) $M_{\lambda, 0}^{1,0}(1,-1 ; b, 0)=S(b) \quad(b \in \mathbb{C}-\{0\})$ [see Aouf [6]].

(2) $M_{\lambda, 0}^{1,1}(1,-1 ; b, 0)=K(b) \quad(b \in \mathbb{C}-\{0\})[$ see Aouf [6]].

(3) $M_{\lambda, 0}^{1,0}(1,-1 ; 1-\alpha, 0)=S^{*}(\alpha)$ for $0 \leq \alpha<1$ [see Wiatrowski [11]].

The classes $S(b)$ and $K(b)$ are said to be the classes of starlike and convex functions of complex order $b \neq 0$ in $\Delta$ which were studied by Nasr and Aouf [6] and Wiatrowski [11] and $S^{*}(\alpha)$ is the class of starlike functions of order $\alpha$ in $\Delta$.

\section{Majorization Problem for the Class $M_{\lambda, m}^{p, j}(A, B ; b, \gamma)$}

Theorem 1. Let the function $f(z)$ be in the class $\mathcal{A}_{p}$ and suppose that $g(z) \in M_{\lambda, m}^{p, j}(A, B ; b, \gamma)$. If $\left(G_{\lambda}^{p, m}\left(\alpha_{1}, \beta_{1}\right) f(z)\right)^{(j)}$ is majorized by $\left(G_{\lambda}^{p, m}\left(\alpha_{1}, \beta_{1}\right) g(z)\right)^{(j)}$ in $\Delta$ for $j \in \mathbb{N}_{0}$, then

$$
\left|\left(G_{\lambda}^{p, m+1}\left(\alpha_{1}, \beta_{1}\right) f(z)\right)^{(j)}\right| \leq\left|\left(G_{\lambda}^{p, m+1}\left(\alpha_{1}, \beta_{1}\right) g(z)\right)^{(j)}\right| \text { for }|z| \leq r_{1},
$$

where $r_{0}=r_{0}(p, b, \lambda, \gamma, A, B)$ is smallest positive root of the equation

$$
\begin{gathered}
|b \lambda(A-B)(1-\gamma)+p B| r^{3}-(p+2 \lambda|B|) r^{2}-(|b \lambda(A-B)(1-\gamma)+p B|+2 \lambda) r+p=0 \\
(-1 \leq B<A \leq 1 ; p \in \mathbb{N}, b \in \mathbb{C}-\{0\})
\end{gathered}
$$


Proof. Let

$$
\begin{aligned}
h(z) & =1+\frac{1}{b}\left(\frac{z\left(G_{\lambda}^{p, m}\left(\alpha_{1}, \beta_{1}\right) f(z)\right)^{(j+1)}}{\left(G_{\lambda}^{p, m}\left(\alpha_{1}, \beta_{1}\right) f(z)\right)^{(j)}}-p+j\right) \\
(p & \left.\in \mathbb{N} ; m, j \in \mathbb{N}_{0} ; b \in \mathbb{C}-\{0\} ; p>j\right) .
\end{aligned}
$$

Since $g(z) \in M_{\lambda, m}^{p, j}(b, \gamma, A, B)$, we find from (1.8) that

$$
h(z)=\frac{1+[(B-A) \gamma+A] w(z)}{1+B w(z)},
$$

where $w(z)$ is analytic in $\Delta$, which satisfies the conditions

$$
w(0)=0 \text { and }|w(z)|<1 \quad(z \in \Delta) .
$$

It follows from (2.3) and (2.4) that

$$
\begin{aligned}
& \frac{z\left(G_{\lambda}^{p, m}\left(\alpha_{1}, \beta_{1}\right) f(z)\right)^{(j+1)}}{\left(G_{\lambda}^{p, m}\left(\alpha_{1}, \beta_{1}\right) f(z)\right)^{(j)}} \\
= & \frac{(p-j)+[b(A-B)(1-\gamma)+(p-j) B] w(z)}{1+B w(z)} .
\end{aligned}
$$

In view of

$$
\begin{aligned}
& \lambda z\left(G_{\lambda}^{p, m}\left(\alpha_{1}, \beta_{1}\right) f(z)\right)^{(j+1)} \\
= & p\left(G_{\lambda}^{p, m+1}\left(\alpha_{1}, \beta_{1}\right) f(z)\right)^{(j)}-(p-p \lambda+\lambda j)\left(G_{\lambda}^{p, m}\left(\alpha_{1}, \beta_{1}\right) f(z)\right)^{(j)}
\end{aligned}
$$

(2.5) immediately yields the following inequality:

$$
\begin{aligned}
& \left|\left(G_{\lambda}^{p, m}\left(\alpha_{1}, \beta_{1}\right) g(z)\right)^{(j)}\right| \\
\leq & \frac{p(1+|B||z|)}{p-|b \lambda(A-B)(1-\gamma)+p B||z|}\left|\left(G_{\lambda}^{p, m+1}\left(\alpha_{1}, \beta_{1}\right) g(z)\right)^{(j)}\right| .
\end{aligned}
$$

Since $\left(G_{\lambda}^{p, m}\left(\alpha_{1}, \beta_{1}\right) f(z)\right)^{(j)}$ is majorized by $\left(G_{\lambda}^{p, m}\left(\alpha_{1}, \beta_{1}\right) g(z)\right)^{(j)}$ in $\Delta$, there exist an analytic function $\phi(z)$

such that

$$
\left(G_{\lambda}^{p, m}\left(\alpha_{1}, \beta_{1}\right) f(z)\right)^{(j)}=\phi(z)\left(G_{\lambda}^{p, m}\left(\alpha_{1}, \beta_{1}\right) g(z)\right)^{(j)}
$$

and $|\phi(z)| \leq 1(z \in \Delta)$. Thuse we have

$$
\begin{aligned}
z\left(G_{\lambda}^{p, m}\left(\alpha_{1}, \beta_{1}\right) f(z)\right)^{(j+1)}= & z \phi^{\prime}(z)\left(G_{\lambda}^{p, m}\left(\alpha_{1}, \beta_{1}\right) g(z)\right)^{(j)} \\
& +z \phi(z)\left(G_{\lambda}^{p, m}\left(\alpha_{1}, \beta_{1}\right) g(z)\right)^{(j+1)} .
\end{aligned}
$$

Using (2.6), in the above equation, we get

$$
\begin{aligned}
\left(G_{\lambda}^{p, m+1}\left(\alpha_{1}, \beta_{1}\right) f(z)\right)^{(j)}= & \frac{\lambda z}{p} \phi^{\prime}(z)\left(G_{\lambda}^{p, m}\left(\alpha_{1}, \beta_{1}\right) g(z)\right)^{(j)} \\
& +\phi(z)\left(G_{\lambda}^{p, m+1}\left(\alpha_{1}, \beta_{1}\right) g(z)\right)^{(j)}
\end{aligned}
$$


Noting that $\phi(z)$ satisfies (cf. [4], [7])

$$
\left|\phi^{\prime}(z)\right| \leq \frac{1-|\phi(z)|^{2}}{1-|z|^{2}} \quad(z \in \Delta),
$$

we see that

$$
\begin{aligned}
& \left|\left(G_{\lambda}^{p, m+1}\left(\alpha_{1}, \beta_{1}\right) f(z)\right)^{(j)}\right| \leq \\
& \left\{|\phi(z)|+\frac{1-|\phi(z)|^{2}}{1-|z|^{2}} \frac{\lambda|z|(1+|B||z|)}{p-|b \lambda(A-B)(1-\gamma)+p B||z|}\right\} \\
& \left|\left(G_{\lambda}^{p, m+1}\left(\alpha_{1}, \beta_{1}\right) g(z)\right)^{(j)}\right|,
\end{aligned}
$$

which, upon setting

$$
|z|=r \quad \text { and }|\phi(z)|=\rho \quad(0 \leq \rho \leq 1)
$$

leads us to the following inequality:

$$
\left|\left(G_{\lambda}^{p, m+1}\left(\alpha_{1}, \beta_{1}\right) f(z)\right)^{(j)}\right| \leq \Psi(r, \rho)\left|\left(G_{\lambda}^{p, m+1}\left(\alpha_{1}, \beta_{1}\right) g(z)\right)^{(j)}\right|,
$$

where

$$
\Psi(r, \rho)=\frac{-\lambda r(1+|B| r) \rho^{2}+\left(1-r^{2}\right) \rho(p-|b \lambda(A-B)(1-\gamma)+p B| r)+\lambda r(1+|B| r)}{\left(1-r^{2}\right)(p-|b \lambda(A-B)(1-\gamma)+p B||z|)} .
$$

In order to determine $r_{1}$, we note that

$$
\begin{aligned}
r_{0} & =\max \{r \in[0,1]: \Psi(r, \rho) \leq 1, \forall \rho \in[0,1]\} \\
& =\max \{r \in[0,1]: \chi(r, \rho) \leq 1, \forall \rho \in[0,1]\}
\end{aligned}
$$

where

$$
\begin{aligned}
\chi(r, \rho)= & \left(1-r^{2}\right)(p-|b \lambda(A-B)(1-\gamma)+p B||z|)- \\
& \rho\left(1-r^{2}\right)(p-|b \lambda(A-B)(1-\gamma)+p B| r)- \\
& \left(1-\rho^{2}\right) \lambda(1+|B| r) r .
\end{aligned}
$$

A simple calculation shows that the inequality $\chi(r, \rho) \geq 0$ is equivalent to

$$
u(r, \rho)=\left(1-r^{2}\right)(p-|b \lambda(A-B)(1-\gamma)+p B||z|)-\lambda(1+|B| r) r(1+\rho) \geq 0 .
$$

Obviously the function $u(r, \rho)$ takes its minimum value at $\rho=1$, i.e.

$$
\min \{u(r, \rho): \rho \in[0,1]\}=u(r, 1)=v(r),
$$

where

$v(r)=|b \lambda(A-B)(1-\gamma)+p B| r^{3}-(p+2 \lambda|B|) r^{2}-(|b \lambda(A-B)(1-\gamma)+p B|+2 \lambda) r+p$ 
It follows that $v(r) \geq 0$ for all $r \in\left[0, r_{0}\right]$, where $r_{0}(p, b, \lambda, \gamma, A, B)$ is the smallest positive real root of the equation (2.2). In fact, as one can see easily, in case, i.e either $|b \lambda(A-B)(1-\gamma)+p B| \neq 0$, or if it is equal to zero, (2.2) has a unique root in the interval $(0,1)$ and this is smallest positive root of equation (2.2). This completes the Theorem 1.

As a special case of Theorem 1 , when $A=1$ and $B=-1$, we have

Corollary 1. [9] Let the function $f(z)$ be in the class $\mathcal{A}_{p}$ and suppose that $g(z) \in M_{\lambda, m}^{p, j}(b, \gamma)$. If $\left(G_{\lambda}^{p, m}\left(\alpha_{1}, \beta_{1}\right) f(z)\right)^{j}$ is majorized by $\left(G_{\lambda}^{p, m}\left(\alpha_{1}, \beta_{1}\right) g(z)\right)^{\prime}$ in $\Delta$ for $j \in \mathbb{N}_{0}$, then

$$
\left|\left(G_{\lambda}^{p, m+1}\left(\alpha_{1}, \beta_{1}\right) f(z)\right)^{j}\right| \leq\left|\left(G_{\lambda}^{p, m+1}\left(\alpha_{1}, \beta_{1}\right) g(z)\right)^{j}\right| \text { for }|z| \leq r_{1},
$$

where

$$
r_{1}=r_{1}(p, b, \lambda, \gamma, A, B):=\frac{k-\sqrt{k^{2}-4 p|p+2 b \lambda(\gamma-1)|}}{2|p+2 b \lambda(\gamma-1)|}
$$

$(k:=2 \lambda+p+|p+2 b \lambda(\gamma-1)| ; p \in \mathbb{N} ; b \in \mathbb{C}-\{0\} ; \lambda \geq 0)$.

Setting $A=1, B=-1, p=1$ and $j=0$ in Theorem 1, we have

Corollary 2. Let the function $f(z)$ be in the class $\mathcal{A}$ be analytic and univalent in the open disk $\Delta$ and suppose that $g(z) \in M_{\lambda, m}^{1,0}(b, \gamma)$. If $\left(G_{\lambda}^{1, m}\left(\alpha_{1}, \beta_{1}\right) f(z)\right)$ is majorized by $\left(G_{\lambda}^{1, m}\left(\alpha_{1}, \beta_{1}\right) g(z)\right)$ in $\Delta$, then

$$
\left|\left(G_{\lambda}^{1, m+1}\left(\alpha_{1}, \beta_{1}\right) f(z)\right)^{\prime}\right| \leq\left|\left(G_{\lambda}^{1, m+1}\left(\alpha_{1}, \beta_{1}\right) g(z)\right)^{\prime}\right| \text { for }|z| \leq r_{2},
$$

where

$$
\begin{aligned}
& r_{2}:=\frac{k-\sqrt{k^{2}-4|1+2 b \lambda(\gamma-1)|}}{2|1+2 b \lambda(\gamma-1)|} \\
& (k:=2 \lambda+1+|1+2 b \lambda(\gamma-1)| ; \quad b \in \mathbb{C}-\{0\} ; \lambda \geq 0) .
\end{aligned}
$$

Further putting $\lambda=1, m=0, q=2, s=1, \alpha_{1}=\beta_{1}$ and $\alpha_{2}=1$ in Corollary 2, we get

Corollary 3. [2] Let the function $f(z)$ be in the class $\mathcal{A}$ be analytic and univalent in the open disk $\Delta$ and suppose that $g(z) \in \varphi(b, \gamma)$. If $f(z)$ is majorized by $g(z)$ in $\Delta$, then

$$
\left|f^{\prime}(z)\right| \leq\left|g^{\prime}(z)\right| \text { for }|z| \leq r_{3}
$$

where

$$
r_{3}=\frac{3+|1+2 b(\gamma-1)|-\sqrt{9+2|1+2 b(\gamma-1)|+|1+2 b(\gamma-1)|^{2}}}{2|1+2 b(\gamma-1)|} .
$$

For $b=1$, Corollary 3 reduces to the following result: 
Corollary 4. [5] Let the function $f(z) \in \mathcal{A}$ be analytic and univalent in the open disk $\Delta$ and suppose that $g(z) \in \varphi^{*}=\varphi^{*}(0)$. If $f(z)$ is majorized by $g(z)$ in $\Delta$, then

$$
\left|f^{\prime}(z)\right| \leq\left|g^{\prime}(z)\right| \text { for }|z| \leq r_{4}
$$

where

$$
r_{4}=\frac{3+|(2 \gamma-1)|-\sqrt{9+2|(2 \gamma-1)|+|(2 \gamma-1)|^{2}}}{2|(2 \gamma-1)|} .
$$

\section{REFERENCES}

[1] F. M. Al-Oboudi, On univalent functions defined by a generalized Salagean operator, Int. J. Math. Math. Sci., no. 25-28, (2004), 1429-1436.

[2] O. Altintas, Ö. Özkan and H. M. Srivastava, Majorization by starlike functions of complex order, Complex Variables Theory Appl. 46, no. 3, (2001), 207-218.

[3] J. Dziok and and H. M. Srivastava, Classes of analytic function associated with the generalized hypergeometric function, Appl. Math. Comput. 103, no. 1, (1999), 1-13.

[4] A. W. Goodman, Univalent functions, Vol. I, Mariner, Tampa, FL, 1983.

[5] T. H. MacGregor, Majorization by univalent functions, Duke Math. J. 34, (1967), 95-102.

[6] M. A. Nasr and M. K. Aouf, Starlike function of complex order, J. Natur. Sci. Math. 25, no. 1, (1985), $1-12$.

[7] Z. Nehari, Conformal mapping, McGraw-Hill, Inc., New york, Toronto, London, 1952.

[8] G. S. Salagean, Subclasses of univalent functions, in Complex analysis-fifth Romanian Finnish seminar, Port 1 (Bucharest), Lecture Notes in Math., 1013, Springer, Berlin, (1981), 362-372.

[9] C. Selvaraj and K. A. Selvakumarn, Majorization problem for certain classes of analytic functions, International Mathematical Forum, Vol. 6, (2011), no. 6, 289 - 294.

[10] C. Selvaraj and K. A. Selvakumarn, Majorization for certain classes of analytic functions defined by a generalized operator, European Journal of Pure and Applied Mathematics, Vol. 3, No. 6, (2010), 1048-1054.

[11] P. Wiatrowski, The coefficients of certain family of holomorphic functions, Zeszyty Nauk. Uniw. Lodz. Nauki Mat. przyrod. Ser. II No. 39 Mat., (1971), 75-85. 